\title{
Breeding of more xylanolytic Butyrivibrio fibrisolvens by electrotransformation
}

\author{
Y Kobayashi, N Okuda, M Wakita, S Hoshino \\ Faculty of Bioresources, Mie University, Tsu, Mie 514, Japan
}

\begin{abstract}
Elevation of cellulase and/or hemicellulase activities is one of the main target in genetic manipulation of rumen bacteria. Using a novel transformation system for Butyrivibrio fibrisolvens, a predominant xylanolytic bacterium in the rumen, we have attempted to modify the species into more xylanolytic organism by heterologous expression of an Eubacterium ruminantium xylanase gene.
\end{abstract}

A xylanase gene from E.ruminantium previously cloned (Kobayashi et al, 1994, Proc Soc Nutr Physiol, 3, 152) was used as a target gene for amplification and expression in B.fibrisolvens. The gene was ligated into PYK4, a shuttle vector between E.coli and B.fibrisolvens. The resultant chimeric plasmid $\mathrm{pYK} 4 \mathrm{X}$ was electroporated into B.fibrisolvens and the transformant was screened by erythromycin resistance, elevated xylanolysis, zymogram and southern analysis. The transformant was employed for determination of growth curve and xylanase activity on various carbon sources.

Transformants obtained at a low frequency $\left(<2 \times 10^{-7}\right)$ were shown to have a high copy number of the target gene on an agarose gel electrophoretogram. The recombinant B.fibrisolvens finally confirmed by southern and zymogram analyses showed 2-3 times higher xylanolytic activity than the parent strain. No significant change in growth rate on xylan was observed after the recombination. However, more accumulation of reducing sugar was notable in the medium of the recombinant. Both the recombinant and parent strains were repressed for their xylanolysis under the presence of glucose, cellobiose or xylose, though the extent of the xylanolysis elevation by the recombination (2-3 times) remained.

Since previous expression of a newly introduced xylanase gene in non-xylanolytic Bacteroides (Whitehead et al, 1991, Appl Environ Microbiol, 57, 277-282) was much greater than in the present study with indigenous xylanolytic B.fibrisolvens, a system regulating xylanase production in the indigenous xylanolytic species should be investigated to promote higher and more stable gene expression. 